\title{
Juxtaglomerular cell tumour of the kidney: a rare cause of resistant hypertension
}

\author{
Nikitas S Skarakis@1, Irene Papadimitriou', Labrini Papanastasiou'1, Sofia Pappa², \\ Anastasia Dimitriadi², Ioannis Glykas ${ }^{3}$, Konstantinos Ntoumas ${ }^{3}$, \\ Penelope Lampropoulou ${ }^{4}$ and Theodora Kounadi ${ }^{1}$
}

1 Unit of Endocrinology and Diabetes Center, 2Department of Pathology, 'G. Gennimatas' General Hospital, Athens, Greece, 3Department of Urology, and 4Department of Radiology, General Hospital of Athens 'G Gennimatas', Athens, Greece
Correspondence should be addressed to N S Skarakis

Email

skar.nik.ns@gmail.com

\section{Summary}

Juxtaglomerular cell tumour (JGCT) is an unusually encountered clinical entity. A 33-year-old man with severe long-standing hypertension and hypokalaemia is described. The patient also suffered from polyuria, polydipsia, nocturia and severe headaches. On admission, laboratory investigation revealed hypokalaemia, kaliuresis, high aldosterone and renin levels, and the abdomen CT identified a mass of $4 \mathrm{~cm}$ at the right kidney. Kidney function was normal. Following nephrectomy, the histological investigation revealed the presence of a JGCT. Immunostaining was positive for CD34 as well as for smooth muscle actin and vimentin. Following surgery, a marked control of his hypertension with calcium channel blockers and normalization of the serum potassium, renin or aldosterone levels were reached. According to our findings, JGCT could be included in the differential diagnosis of secondary hypertension as it consists of a curable cause. The association of JGCT with hypertension and hypokalaemia focusing on the clinical presentation, diagnostic evaluation and management is herein discussed and a brief review of the existing literature is provided.

\section{Learning points:}

- Juxtaglomerular cell tumours (JGCT), despite their rarity, should be included in the differential diagnosis of secondary hypertension as they consist of a curable cause of hypertension.

- JGCT could be presented with resistant hypertension along with hypokalaemia, kaliuresis and metabolic alkalosis. Early recognition and management can help to prevent cardiovascular complications.

- Imaging (enhanced CT scans) may be considered as the primary diagnostic tool for the detection of renal or JGCT.

- For the confirmation of the diagnosis, a histopathologic examination is needed.

\section{Background}

Juxtaglomerular cell tumour (JGCT), or reninoma as first reported by Robertson in 1967 and Kihara in 1968, consists of a tumour derived from the afferent arteriole of the juxtaglomerular apparatus of the kidney (1). It comprises a rare cause of secondary hypertension, usually severe and drug-resistant, accompanied in the majority of cases with secondary hyperaldosteronism and profound hypokalaemia. JGCT is characterized by female predominance with a peak occurrence in the second and third decades (2). While this tumour is considered benign, cases with malignant potential with metastatic lesions and vascular invasion have been described (2).

Herein, we report a case of a young man presented with a long history of malignant hypertension along with hypokalaemia, secondary to aJGCT. We discuss the patient's clinical presentation, diagnostic evaluation, management and follow-up. To the best of our knowledge, this is the first case of JGCT with typical characteristics reported in Greece. 


\section{Case presentation}

A 33-year-old Greek man was referred to the Endocrinology Unit of a tertiary care hospital for the diagnostic evaluation and treatment of hypertension despite receiving antihypertensive drugs. His medical history revealed that the patient was suffering from malignant hypertension since the age of 28 years accompanied by severe headache with migraine, polydipsia, polyuria, nocturia and dizziness, without a history of visual disturbances. He had a long family history of hypertension, while his brother suffered from myocardial infarction at a young age (35 year-old-age). His only medication was a calcium channel blocker for high blood pressure and an over-the-counter analgesic for his headaches. He denied any history of tobacco or alcohol abuse.

\section{Investigation}

On admission, blood pressure values at $210 / 120 \mathrm{mmHg}$ with regular pulses were confirmed. Cardiovascular examination was unremarkable. Left ventricular ejection fraction was exceeded to $60 \%$. Fundoscopy was indicative of hypertensive retinopathy.

In order to exclude other causes of headache, brain MRI and brain angiography were conducted without abnormal findings.

Biochemical evaluation showed marked hypokalaemia ranging from 2.4 to $3.0 \mathrm{mEq} / \mathrm{L}$ (NR: $3.2-4.8 \mathrm{mEq} / \mathrm{L}$ ) requiring i.v. infusion of potassium, and urinalysis showed marked proteinuria $(1.89 \mathrm{mg} / 24 \mathrm{~h}, \mathrm{NR}:<0.19 \mathrm{mg} / 24 \mathrm{~h})$. Twenty-four-h urine collection revealed normal sodium excretion (185 mEq, NR: 40-220 mEq/24 h) with elevated potassium excretion (276 mEq/24 h, NR: $25-125 \mathrm{mEq} / 24$ h). Renal function was within normal range with creatinine levels at $0.9 \mathrm{mg} / \mathrm{dL}$ (NR: $0.7-1.3 \mathrm{mg} / \mathrm{dL}$ ) and estimated GFR at $103.7 \mathrm{~mL} / \mathrm{min} / 1.73 \mathrm{~m}^{2}$.

Hormonal investigations showed extremely elevated both direct renin concentration (DRC) and aldosterone at $352 \mathrm{pg} / \mathrm{mL}$ (NR: 2.7-16.5 pg/mL) (587.84 mU/L, NR: 4.527.5) and $1114 \mathrm{pmol} / \mathrm{L}$ (NR: 137-486 pmol/L), respectively.
The patient underwent evaluation with imaging studies to localize the source of renin overproduction. Abdominal ultrasonography revealed a mass of $4 \mathrm{~cm}$ of the right kidney and abdomen CT demonstrated a solid mass of $4.5 \mathrm{~cm}$ at the upper pole of the right kidney and duplication of the right renal vein (Fig. 1A and B). Computed tomographic angiography (CTA) was not indicative of renal artery stenosis (Fig. 1A). Drug-resistant hypertension, hypokalaemia, kaliuresis, along with increased DRC and aldosterone and a kidney mass of 4.5 $\mathrm{cm}$ led to the diagnosis of secondary hyperaldosteronism probably due to a JGCT.

\section{Treatment}

On admission, hypertension was treated with a combination of a Calcium Channel Blocker (CCB) (diltiazem), along with a centrally acting antihypertensive drug (moxonidine), a mineralocorticoid receptor antagonist (eplerenone) with a moderate improvement of his blood pressure. Following the identification of a tumour in the right kidney, an open right total nephrectomy was performed in the patient. The patient had an uneventful postoperative course. Blood pressure as well as potassium levels normalized after surgery; hence, potassium supplements and antihypertensive drugs were discontinued. However, on the second postoperative day, the patient developed hypertension (BP: 155/100 $\mathrm{mmHg}$ ), so a CCB was restarted. Furthermore, polyuria, nocturia and polydipsia disappeared immediately after surgery.

Histological results confirmed the diagnosis of JGCT consisting of a $3.7 \mathrm{~cm}$ encapsulated, well-circumscribed tumour, with cystic and hemorrhagic changes, dilated and entrapped renal tubules confined to the kidney without involvement of the perinephric or sinus fat (Fig. 2A). Light microscopy showed that the tumour consisted of clusters of polygonal cells and the cell had centrally located nuclei and slightly eosinophilic cytoplasm (Fig. 2B). The tumour did not show nuclear pleomorphism or marked mitotic activity. Immunostaining was positive for CD34 (Fig. 2C
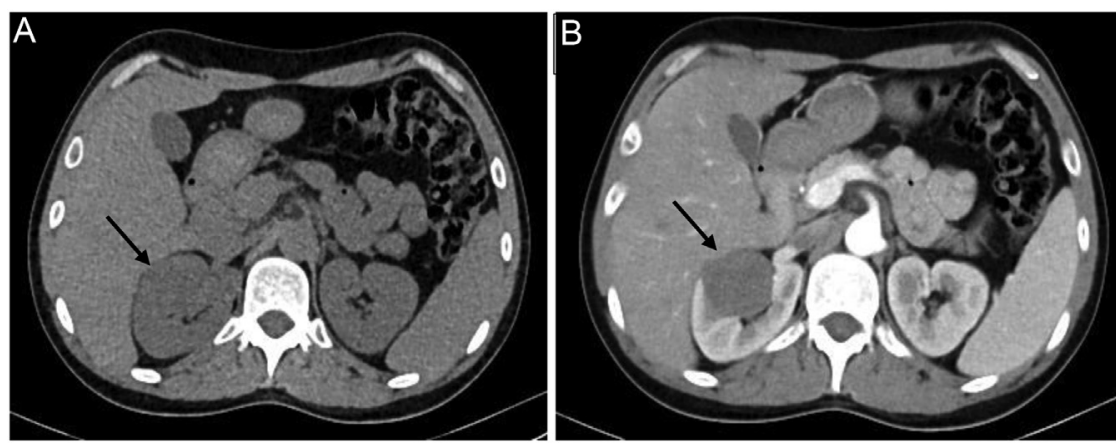

Figure 1

Dynamic CT of juxtaglomerular cell tumour of the kidney. (A) In plain scan, the tumour appeared isodense to the renal medulla. (B) In contrast enhancement, the tumour was hypodense compared to the renal medulla. 
and D) as well as for smooth muscle actin (SMA) and vimentin but was negative for CD31 and WT-1.

\section{Outcome and follow-up}

Renin, aldosterone and potassium levels normalized immediately postoperatively. Follow-up examinations at 3 and 6 months showed a control of his blood pressure with $\mathrm{CCB}$, while no abnormalities in the serum potassium, renin or aldosterone levels were established (Table 1).

Written informed consent was obtained from the patient for publication of this case and accompanying images.

\section{Discussion}

In this report, we present an unusual case of long-standing severe hypertension, hypokalaemia and high aldosterone and renin levels that was attributed to a JGCT of the right kidney. Since the tumour was large and deep, the patient underwent open radical right nephrectomy without any complication. Serum and urinary potassium levels as well as renin and aldosterone levels have returned to normal, and blood pressure was well controlled with a calcium channel blocker.

Since 1967 when the first case of reninoma or JGCT was described, approximately 165 cases have been referred in the literature. The mean age of diagnosis was 27.9 years with a female to male ratio 1.8:1 (2). However, in a group of cases, the diagnosis of JGCT regarded children under 16 years old with four of them being under 10 years old (2). Indeed, one of the youngest cases reported in the literature was an 8-year-old boy referred as the only one with multicentric origin (3). Furthermore, JGCT has also been described during pregnancy with various outcomes, either fetal loss due to severe hypertension in most of the cases or normal labour without complications (4).

JGCTconstitutesabenigntumourofthejuxtaglomerular apparatus characterized by the overproduction of renin and secondary hyperaldosteronism. However, since today, five cases with malignant characteristics have been
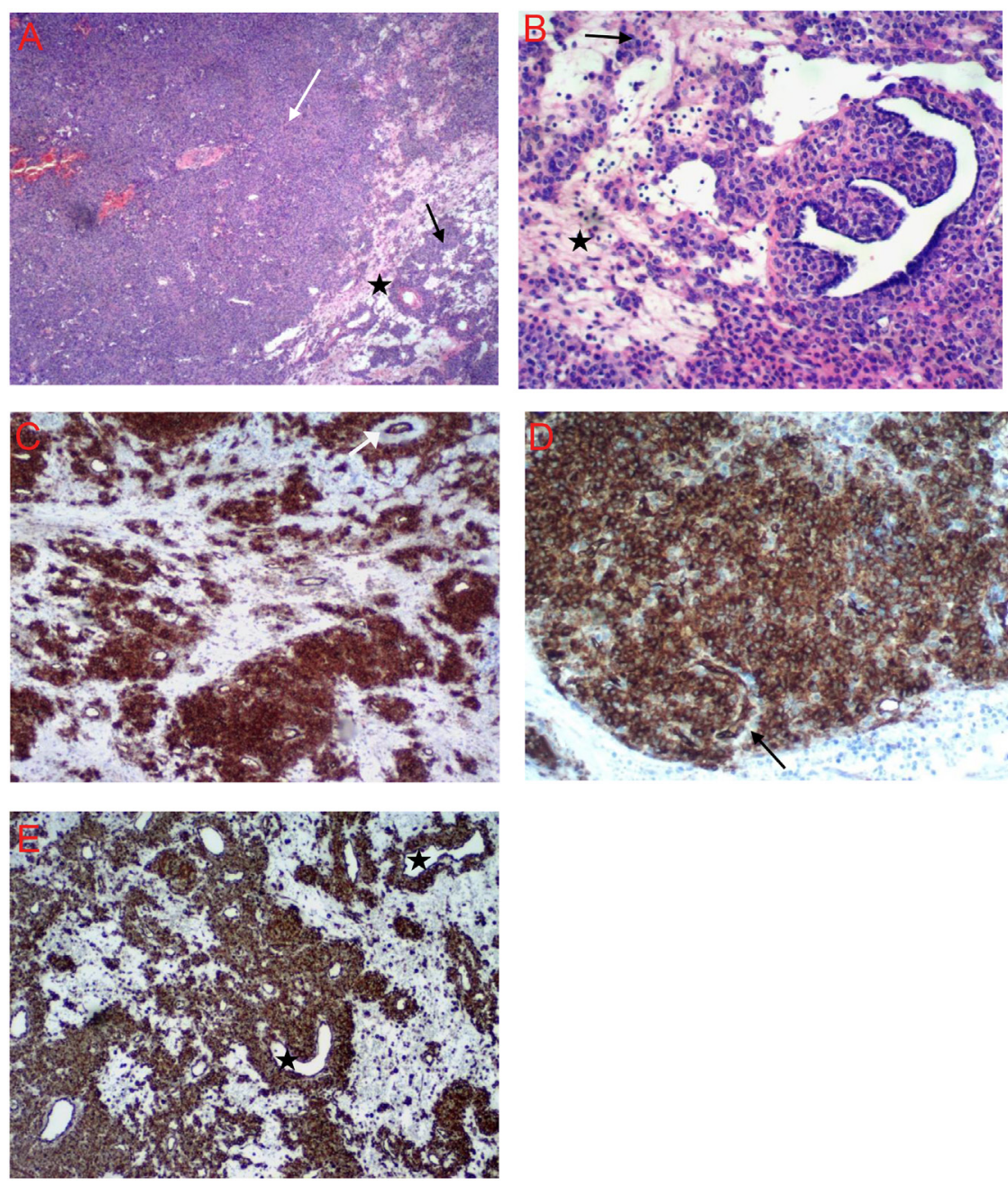

\section{Figure 2}

Histopathology of reninoma. (A) Haematoxylin and eosin, $\times 20$ magnification. (B) Haematoxylin \& eosin, $\times 100$ magnification. The tumour is composed of generally uniform polygonal to spindle cells with eosinophilic cytoplasm arranged in sheets (A) and trabeculae (arrows in $A$ and $B$ ). The stroma is generally oedematous $(\star)$ and focally scant (white arrow). (C and D) CD34 immunostain, $\times 50$ and $\times 100$ magnification, respectively. The tumour cells stain positive for CD34. The tumour shows prominent hemangiopericytoma-like vasculature (black arrow), often with gaping vessels. Adjacent vasculature (white arrow) acts as an internal positive control. (E) Vimentin immunostain $\times 50$ magnification. The neoplastic cells are positive for vimentin. The vessels inside the tumour can sometimes appear gaping $(\star)$. 
Table 1 Hormonal and biochemical parameters before and after surgery.

\begin{tabular}{l}
\hline \\
\hline Renin \\
Renin (pg/ml) \\
Aldosterone \\
Sodium \\
Potassium
\end{tabular}

\begin{tabular}{c}
\hline Before \\
\hline 352 \\
587.84 \\
1114 \\
132 \\
2.4 \\
\hline
\end{tabular}

\begin{tabular}{c}
\hline First postoperative day \\
\hline 18.6 \\
31.06 \\
325 \\
133 \\
4.6 \\
\hline
\end{tabular}

\begin{tabular}{c}
\hline Thirdpostoperative day \\
\hline 13.6 \\
22.71 \\
233 \\
139 \\
4.1
\end{tabular}

\begin{tabular}{c}
\hline 3 months \\
\hline 11.5 \\
19.20 \\
302 \\
140 \\
4.0 \\
\hline
\end{tabular}

\begin{tabular}{c}
\hline $\mathbf{6}$ months \\
\hline 10.2 \\
17.03 \\
295 \\
140 \\
4.2 \\
\hline
\end{tabular}

\begin{tabular}{c}
\hline Normal values \\
\hline $2.71-16.51 \mathrm{pg} / \mathrm{mL}$ \\
$4.5-27.5 \mathrm{mU} / \mathrm{L}$ \\
$137.05-486.5 \mathrm{pmol} / \mathrm{L}$ \\
$135-144 \mathrm{mEq} / \mathrm{L}$ \\
$3.2-4.8 \mathrm{mEq} / \mathrm{L}$
\end{tabular}

described. Their malignant potency was confirmed either by metastases in solid organs (liver and lungs) or by tumour recurrence and vascular invasion on histopathology (5). Furthermore, in one of these cases, the patient presented with paraneoplastic syndrome. Indeed, the patient at the time of diagnosis was presented with hypotonic euvolemic hyponatremia indicative of the syndrome of inappropriate secretion of antidiuretic hormone, with recurrent episodes of hyponatremia (6).

JGCT is mainly presented with malignant hypertension usually resistant to antihypertensive drugs. Long-standing hypertension may lead to congestive heart failure especially in association with ventricular hypertrophy and preserved systolic function. Other symptoms commonly presented were headache, polyuria, nocturia, excessive thirst, dizziness and fatigue. The same symptoms were present in our patient. Retinopathy has been reported in $17 \%$ of previously reported cases (7).

Autonomous secretion of renin from juxtaglomerular cells of the tumour results in the activation of reninangiotensin-aldosterone system (RAAS). Excess aldosterone synthesis and secretion from adrenal glands following RAAS activation stimulates sodium-potassium exchange by the principal cells of the collecting duct leading to excessive potassium loss in urine, depletion of body potassium and stimulation of proton secretion by the kidney intercalated cells of the collecting duct, with the development of hypokalaemia and metabolic alkalosis. Differential diagnosis should encompass causes of secondary hypertension, including several other reninproducing tumours other than JGCT that are listed in Table 2 (5). The most common causes of secondary hypertension include primary renal or renovascular disease (renal artery stenosis), sleep apnea syndrome, congenital or acquired cardiovascular disease (i.e. coarctation of the aorta), oral contraceptive use and excess hormonal secretion. The most common causes of endocrine hypertension are of adrenal origin due to excess production of mineralocorticoids (i.e. primary hyperaldosteronism), glucocorticoids (Cushing syndrome) and catecholamines (pheochromocytoma).

Primary renal or renovascular disease is the most common cause of secondary hypertension in the young and the second most common cause in adult patients. Urine analysis and measurement of serum creatinine concentration are the best screening methods. If they are abnormal, a renal ultrasound should be the next step. Further workup includes CT or MRI imaging. If significant renal artery stenosis is suspected, renal angiography with hemodynamic assessment should be considered. A history of snoring and daytime sleepiness should prompt to suspect of sleep apnea syndrome and assess sleepiness using the Epworth Sleepiness Scale. When hypertension is associated with hypokalaemia and metabolic acidosis, primary aldosteronism is suspected and the plasma aldosteronerenin ratio should be assessed as a first screening step.

At 2010, Dong et al. proposed a new classification for JGCT according to the clinical presentation and the laboratory examination. As though, the patients can be classified into three types: the typical type (hypertension, hyperaldosteronism and hypokalaemia secondary to tumour renin secretion), the atypical type (hypertension with normal potassium) and the non-functioning type (normal blood pressure and normal potassium). Our patient followed the typical pattern. According to this classification, 12 of the 71 cases have been reported as atypical, 2 as nonfunctioning and 57 had the typical presentation (8).

Enhanced CT scans are the imaging study of choice for all suspected JGCT cases that appear as small isodense lesions which may not be detected by unenhanced CT scans. They are particularly sensitive (100\%) in detecting this kind of tumour compared to MRI scans (4). On renal angiography, the tumour appears as a small avascular area (false-negative results in about $43 \%$ of cases), and it allows for the exclusion

Table 2 Renin-producing tumours in the differential diagnosis for JGCT.

\begin{tabular}{|c|c|}
\hline Renin-producing tumours & Prevalence \\
\hline B-cell leukaemia & $1-5 / 10000$ \\
\hline Breast carcinoma & $25.1 \%$ \\
\hline Cystadenocarcinoma & $2 \%$ \\
\hline $\begin{array}{l}\text { Desmoplastic small round cell } \\
\text { tumour }\end{array}$ & 0.3 cases/million \\
\hline Hepatoblastoma & $2.16 / 1000000$ \\
\hline Wilm's tumour & $1-9 / 100000$ \\
\hline Uterine leiomyosarcoma & $0.35-0.64 / 100000$ \\
\hline
\end{tabular}


of renal arterial stenosis, as a potential cause of secondary hyperaldosteronism (9). In some cases, though, it is not always easy to be detected as these tumours are usually small and superficially located in the renal cortex.

Renal venous sampling may be useful in small tumours or in cases where no tumour can be detected with the aforementioned imaging studies. A plasma renin activity lateralizing ratio of at least 1.5 is usually diagnostic for JGCT with a sensitivity of $56 \%$ and specificity of $94 \%$ (9). Therefore, due to the low positive rate and the invasive nature of the test, it is not recommended as mandatory in the diagnosis of JGCT. As imaging studies detected the tumour, we did not proceed to this test in our patient.

Histopathological analysis plays a crucial role in the definite diagnosis of JGCT. The pathologic features of JGCT include actin and CD34 positivity, along with cytokeratin negativity. These markers in conjunction with vimentin and SMA positivity are recommended for the diagnosis of JGCT and the differentiation from renal cell carcinoma. Other characteristic features include the presence of rhomboid renin granules on ultrastructural studies (10).

As far as the management of JGCT is concerned, nephron-sparing surgery remains the procedure of choice with partial nephrectomy or tumour resection. Laparoscopic approach is mainly recommended as the tumours are usually small and superficial and consequently easily accessible. However, in cases of large or deep, centrally located tumours in which nephron-sparing surgery is not feasible, radical nephrectomy is recommended for a complete cure (5). Besides the surgical approach, in some patients who were unwilling or unsuitable for surgery or before the surgical approach, pharmacologic management of JGCT with a renin inhibitor, aliskiren, has been proved to be beneficial.

In conclusion, JGCT is suggested to be considered in the differential diagnosis of secondary hypertension as it consists of a curable cause. Combination of a careful clinical evaluation with laboratory and imaging studies are the key factors in suspected cases, with histopathologic examination being mandatory for the confirmation of the diagnosis.

\section{Declaration of interest}

The authors declare that there is no conflict of interest that could be perceived as prejudicing the impartiality of the research reported.

\section{Funding}

This work did not receive any specific grant from any funding agency in the profit, commercial or not-for-profit sector.

\section{Patient consent}

The patient has submitted written informed consent to publish this observation.

\section{Author contribution statement}

All the authors made a major contribution to the patient's clinical care and the intellectual content of this case report.

\section{References}

1 Kihara I, Kitamura S, Hoshino T, Seida H \& Watanabe T. A hitherto unreported vascular tumor of the kidney: a proposal of 'juxtaglomerular cell tumor'. Acta Pathologica Japonica 196818 197-206. (https://doi.org/10.1111/j.1440-1827.1968.tb00048.x)

2 Trnka P, Orellana L, Walsh M, Pool L \& Borzi P. Reninoma: an uncommon cause of renin-mediated hypertension. Frontiers in Pediatrics 20142 89. (https://doi.org/10.3389/fped.2014.00089)

3 Shera AH, Baba AA, Bakshi IH \& Lone IA. Recurrent malignant juxtaglomerular cell tumor: a rare cause of malignant hypertension in a child. Journal of Indian Association of Pediatric Surgeons 201116 152-154. (https://doi.org/10.4103/0971-9261.86876)

4 Xue M, Chen Y, Zhang J, Guan Y, Yang L \& Wu B. Reninoma coexisting with adrenal adenoma during pregnancy: a case report. Oncology Letters 201713 3186-3190. (https://doi.org/10.3892/ol.2017.5802)

5 Inam R, Gandhi J, Joshi G, Smith NL \& Khan SA. Juxtaglomerular cell tumor: reviewing a cryptic cause of surgically correctable hypertension. Current Urology 201913 7-12. (https://doi. org/10.1159/000499301)

6 Cucchiari D, Bertuzzi A, Colombo P, De Sanctis R, Faucher E, Fusco N, Pellegrinelli A, Arosio P \& Angelini C. Juxtaglomerular cell tumor: multicentric synchronous disease associated with paraneoplastic syndrome. Journal of Clinical Oncology 201331 e240-e242. (https://doi. org/10.1200/JCO.2012.43.5545)

7 Beevers DG, Maheshwari MB, Ryan PG, Moss MS \& Harding LK. Hypertension due to a renin-secreting juxtaglomerular cell tumor. American Journal of Hypertension 200821 1359-1361. (https://doi. org/10.1038/ajh.2008.281)

8 Dong D, Li H, Yan W \& Xu W. Juxtaglomerular cell tumor of the kidney - a new classification scheme. Urologic Oncology 201028 34-38. (https://doi.org/10.1016/j.urolonc.2009.08.003)

9 Wong L, Hsu TH, Perlroth MG, Hofmann LV, Haynes CM \& Katznelson L. Reninoma: case report and literature review. Journal of Hypertension 200826 368-373. (https://doi.org/10.1097/ HJH.0b013e3282f283f3)

10 Wang F, Shi C, Cui Y, Li C \& Tong A. Juxtaglomerular cell tumor: clinical and immunohistochemical features. Journal of Clinical Hypertension 201719 807-812. (https://doi.org/10.1111/jch.12997)

Received in final form 5 September 2021 Accepted 21 December 2021 